\title{
Thoughts on cartilage tissue engineering: A 21st century perspective
}

\author{
Theofanis Stampoultzis ${ }^{1}$, Peyman Karami ${ }^{1}$, Dominique P. Pioletti* \\ Laboratory of Biomechanical Orthopedics, EPFL, Lausanne, Switzerland
}

\section{A R T I C L E I N F O}

\section{Article history:}

Received 9 October 2020

Accepted 26 May 2021

Available online 27 June 2021

\section{Keywords:}

Cartilage regeneration

Mechanobiology

Bioreactors

Clinical translation

\begin{abstract}
A B S T R A C T
In mature individuals, hyaline cartilage demonstrates a poor intrinsic capacity for repair, thus even minor defects could result in progressive degeneration, impeding quality of life. Although numerous attempts have been made over the past years for the advancement of effective treatments, significant challenges still remain regarding the translation of in vitro cartilage engineering strategies from bench to bedside. This paper reviews the latest concepts on engineering cartilage tissue in view of biomaterial scaffolds, tissue biofabrication, mechanobiology, as well as preclinical studies in different animal models. The current work is not meant to provide a methodical review, rather a perspective of where the field is currently focusing and what are the requirements for bridging the gap between laboratory-based research and clinical applications, in light of the current state-of-the-art literature. While remarkable progress has been accomplished over the last 20 years, the current sophisticated strategies have reached their limit to further enhance healthcare outcomes. Considering a clinical aspect together with expertise in mechanobiology, biomaterial science and biofabrication methods, will aid to deal with the current challenges and will present a milestone for the furtherance of functional cartilage engineering.
\end{abstract}

(C) 2021 The Authors. Published by Elsevier Masson SAS.

This is an open access article under the CC BY license (http://creativecommons.org/licenses/by/4.0/)
Impact statement Highlights

The worldwide prevalence of cartilage disorders has trended steeply upward and it is assumed to double by 2040. In this context, lack of appropriate strategies to rejuvenate cartilage function, has brought a colossal amount of effort in this expanding field of tissue engineering of effective cartilage tissue analogues. Even though there are multiple reviews available revolved around cartilage regeneration, there is an apparent lack of review papers that highlight not only the scientific requirements, but also the considerations regarding the clinical translation of cartilage engineering strategies. Accordingly, this paper attracts the interest of a broader scientific audience.

\footnotetext{
Author contribution: T.S. and P.K. performed the literature search, prepared the manuscript and figures. D.P.P. edited the review and supervised the preparation of the manuscript.

Declaration of Competing Interest: No competing financial interests exist.

^ Acknowledgments: This work was supported by a SNF grant \# CR23I3_159301.

* Corresponding author.

E-mail addresses: theofanis.stampoultzis@epfl.ch (T. Stampoultzis), peyman.karami@epfl.ch (P. Karami), dominique.pioletti@epfl.ch (D.P. Pioletti).

1 Both the authors have equal contribution to this work.
}

\section{Introduction}

Cartilage injuries are of great clinical consequence, due to the tissue's limited regenerative capacity [1,2]. Today, the currently major repair strategies are still unsatisfactory, mainly because of the fact that they rarely return the tissue to its healthy state or restore its function [3]. Although initially viewed as a tissue with a simple architecture, reproducing the exquisitely stratified structure has proven to be a hard task [4]. Recently, developments in the field of Tissue Engineering have provided alternative options for restoring cartilage anatomical structure.

Indeed, the last 20 years have borne evidence to an enormous expansion in biomaterial science, mechanobiology and manufacturing processes, that could potentially have a beneficial impact on cartilage regeneration. In terms of biomaterials, advances involve a wide array of formulations, spanning from the use of biostable/inert [5] and degradable polymers [6], to the present-day application of third-generation hydrogels with advanced chemistries, aiming to stimulate the process of natural tissue regeneration [7-9]. From a mechano-biological point of view, the framework established by the role of mechanical loading in cartilage homeostasis, resulted in the development of novel bioreactor systems, providing the necessary means for studying chondrocyte mechanotransduction $[10,11]$. In addition to this mate- 
rial and mechanobiological frame, developments in material processing also added a useful tool towards the fabrication of more biomimetic cartilage-like substitutes [12].

Indeed, engineering scalable cartilage constructs through a combination of biomaterial scaffolds, cells and incorporation of appropriate mechanical stimuli will undoubtedly result in incessant opportunities for cartilage repair and regeneration. The work conducted by Christopher O'Conor and colleagues for instance, exemplifies that high-quality cartilaginous tissue can be formed via signaling pathways through activation of transient receptor ion channels under dynamic stimulation [13]. At the same time, biofabrication strategies allow tissue engineers to develop highly-organized constructs that can better facilitate chondrogenesis, in terms of gene expression, matrix synthesis and biomechanical functionality, under a physiologically relevant loading regime. In this regard, a lot of effort has been made in creating soft network constructs that closely resemble natural soft biological materials, such as cartilage, by means of design and composition, as well as physicochemical properties [14-16]. However, despite such remarkable achievements, great challenges still exist when it comes to the translation from research to products.

This review paper intends to highlight the scientific research requirements along with the considerations regarding the clinical translation of cartilage engineering strategies. The objective of this paper is to present and comment on these requirements and not to provide an extensive review of each angle of the field. Therefore, this review will try to discuss the scientific cartilage tissue engineering concepts and will also provide a perspective on how to bridge the gap between cartilage research and clinical applications.

\section{Brief insights in articular cartilage biology and mechanical function}

Articular cartilage covers the gliding surfaces of synovial joints. The architecture of hyaline cartilage provides unique functional characteristics including low friction articulation and damping the mechanical loads in the joint $[17,18]$. This avascular and aneural hyaline cartilage is composed of highly specialized chondrocytes cells, which produce and maintain the dense cartilage extracellular matrix (ECM). Collagen is the major matrix macromolecule consisting $10-20 \%$ of the cartilage weight. Both the compressive resistance and tensile resilience of this soft tissue can be ascribed to this highly organized collagen network $[19,20]$. Proteoglycans such as aggrecan, present another important structural component of this tissue. They make up $10-15 \%$ weight of articular cartilage and they further increase cartilage compressive strength in terms of their fixed charge density and high osmotic pressure [21].

High water-containing environment (65-80\%) of cartilage is retained by the matrix components [22]. The presence of water, together with the collagen network and the various glycoproteins attribute a biphasic nature to the tissue [23]. A fluid phase, mainly consisted of ions dissolved inside water, and a solid permeable matrix containing a network of collagen type II and proteoglycan agglomerations, result in a predominantly viscoelastic behavior. When the tissue is under mechanical loading, the aforementioned viscoelastic characteristics are further enhanced, due to the exudation of the interstitial fluid through the porous solid matrix of the tissue [24].

Based on the unique macrostructure, cartilage tissue is composed of four regions: superficial, middle, deep, and calcified zones; each one exhibiting unique structural characteristics. Such a zonal inhomogeneity, results in a depth-dependent variation of its mechanical properties [25]. Indeed, Antons et al., showed that from the superficial layer of cartilage towards the calcified zone, a gradient of elastic modulus values spanning from $0.02 \mathrm{MPa}$ to approximately 6.44 MPa is evident [26]. Taken together, it can be realized that permeability, viscoelasticity, non-linearity and inhomogeneity present the 4 key mechanical characteristics of articular cartilage.

\section{Cartilage repair strategies over the last 20 years}

Cartilage stems from the Latin word "cartilago", which has the meaning of "chondros" in Greek [27]. Indeed, the first documented realization of cartilage as an indispensable tissue in the human body, has been ascribed to Aristotle in the fourth century BC [28]. Another renowned anatomist, John Hunter (1743) stated that: "An ulcerated cartilage is an intractable disease and once injured is hardly recovered" [29]. This famous quote remained a general belief, regarding cartilage repair, for approximately twohundred years. Indeed, the quest for the most effective way to restore cartilage malfunctions was initiated in the early 1980s [30]. Over the last 30 years, several cartilage repair strategies have been developed for the treatment of such lesions. Among these strategies, contemporary clinical treatments for reconstructive cartilage surgery comprise bone marrow stimulation techniques, autologous chondrocyte implantation and osteochondral autograft and allograft transplantation [31]. A graphical illustration of the aforementioned strategies is depicted in Fig. 1. A brief description of each method is also provided in the following sub-sections.

\section{Marrow stimulation strategies}

Marrow stimulating strategies principally involve debridement of injured cartilage, which result in bleeding, with the goal of inducing healing from the bone marrow. Microfracture surgery presents the "gold standard" among the different marrow stimulating strategies. This technique relies on the body's own healing capabilities and aims to augment chondral resurfacing by providing enhanced milieu for tissue regeneration. In the first step, the calcified layer of cartilage is removed using a curette. A sharp arthroscopic awl is subsequently used to create small fractures (holes of $2 \mathrm{~mm}$ ) in the subchondral bone plate, aiming to induce clot formation in the defected area [32]. Microfracture presents a relatively inexpensive surgical procedure, it provides immediate relief of symptoms, but the durability of sustained clinical improvement beyond 2 years following surgery is debatable. Patients treated with microfracture, for instance, have shown initial clinical improvements in the first months following surgery, however a dramatic decrease in ICRS and Tegner scores was observed after long-term follow-up studies [33]. To address such issues, "enhanced" or in other words "augmented" marrow stimulating methods were proposed. These mainly involve the use of an acellular bio-membrane together with stimulating molecules that aim to prevent cell loss from the clot [34]. However, up to now, there are no studies that convincingly demonstrate the supremacy of such strategies over conventional microfracture.

\section{Osteochondral autograft and allograft transplantation}

Osteochondral autograft transfer, or in other words known as mosaicplasty, involves acquiring cylindrical cartilage-bone plugs from areas that bear minimal loads (such as femoral condyles), and transfering them to the injured sites on the weight-bearing regions [35]. This approach is often recommended for the treatment of medium-size defects and combinations of different graft sizes can allow for a filling rate up to almost 95\%. Limited graft availability presents the main disadvantage of this method and this latter has led to the exploration of other strategies for the treatment of cartilage defects [31]. 

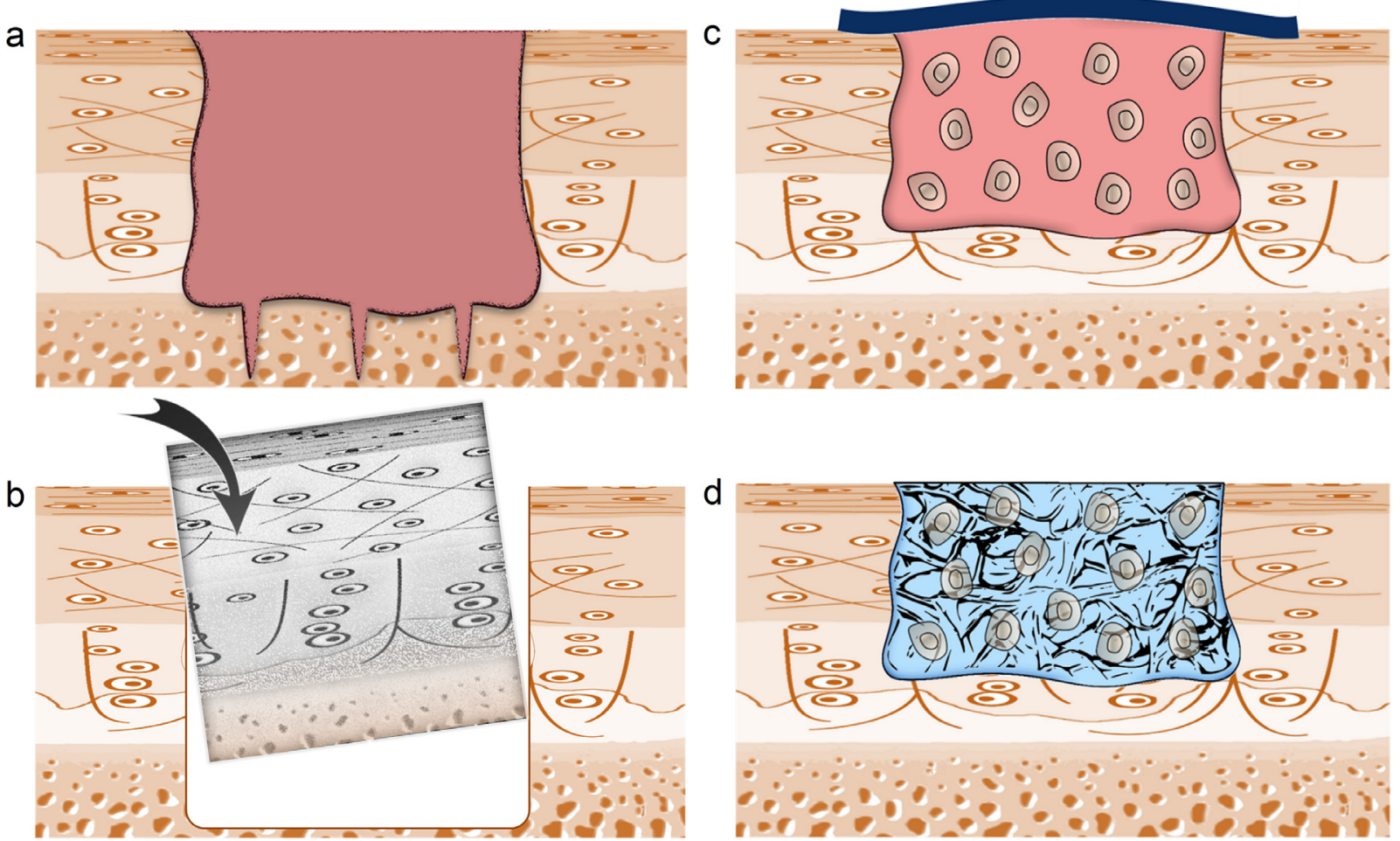

Fig. 1. Current strategies for the treatment of cartilage injuries.

The rationale behind osteochondral allograft transplantation for instance, centers around the transplantation of full-thickness hyaline articular cartilage into the defected area in the joint. It presents a single-stage procedure that limits donor site morbidity and results in more physiologically matching profile of the native recipient surface anatomy [36]. This latter leads to high degrees of long-term implant survival and high rates of reported patient satisfaction. In fact, a 2015 study demonstrated that the survivorship of osteochondral allografts was more than $80 \%$ at 10 years following transplantation, as indicated, mainly, by the Knee injury and Osteoarthritis Outcomes Score (KOOS score) and the Knee Society Function scale (KS-F) [37]. Another elegant study illustrated that among patients who were treated with osteochondral allograft transplantation, more than $85 \%$ showed good or even excellent clinical outcomes at 10 years after surgery [38].

\section{Autologous chondrocyte implantation and matrix assisted autologous chondrocyte implantation}

Autologous chondrocyte implantation presents a two-stage strategy for cartilage repair. The first procedure is performed arthroscopically, where the size of the lesion is assessed. A piece of articular cartilage is then harvested and the cartilage biopsy is enzymatically treated in order to isolate the chondrocytes. Once the chondrocytes are obtained, they are usually expanded in vitro for approximately 6-8 weeks. After this stage, the second procedure begins. More specifically, the operation involves a procedure where a periosteal flap is sewn over the defect. The expanded chondrocytes from stage one, are then injected underneath the patch where they adhere to the patient's knee and they will eventually produce hyaline-based cartilage [39].

More recently, matrix assisted autologous chondrocyte implantation (MACI), involves resuspension of the cells into a hydrated matrix, before injected under the lesion. This technique is mainly used for the treatment of full-thickness cartilage defects and it has shown superior long-term outcomes compared to ACI. Patients who have underwent MACI surgical treatment have demonstrated considerable enhancements in knee joint function [40]. A 2017 study for example, showed that more than $90 \%$ of patients were satisfied with their pain relief at 5 years after surgery and they were able to undertake daily activities, while around $80 \%$ were able to participate again in sports [40].

\section{Cell-based tissue engineering products for cartilage repair}

All in all, the approaches that have been pursued over the years for the treatment of defects in joint cartilage (and were described up to Section 3.3) exhibit limitations. Regenerative strategies (i.e., $\mathrm{ACI} / \mathrm{MACI}$ ) produces more favorable outcomes for the cure of large defects compared to marrow stimulation approaches [41]. However, such outcomes are palliative only, providing temporary relief and no actual treatment, since the structural coherence of the tissue surface is inferior compared to the native tissue [42]. An unmet need for cartilage tissue engineering rises from the tissue's incapacity to mount an adequate healing response after injury. This brief section aims to inform the reader about some of the major and relatively recent clinical applications for articular cartilage repair products in the clinical pipeline. Only cellular constructs are presented.

Aiming to boost cartilage regeneration, a number of cellbased, cartilage products have entered clinical trials around the world. NOVOCART® 3D, a collagen type 1 based scaffold seeded with primary chondrocytes, has been successfully used in Europe for the treatment of more than 6000 patients since 2003 [43]. At the same time a Phase III trial comparing the superiority of NOVOCART ${ }^{\circledR} 3 \mathrm{D}$ over microfracture surgery with 233 participants in total, is expected to be completed in August 2026 [43]. REVAFLEX ${ }^{\mathrm{TM}}$ presents a "scaffoldless" approach where a cartilage-disk is matured from allogeneic juvenile chondrocytes [44]. In a Phase I/II study, $67 \%$ of the patients that were treated with REVAFLEX ${ }^{\mathrm{TM}}$, showed normal or nearly normal repair of the initially injured site [44]. CARTISTEM, a hyaluronic acid-based hydrogel, seeded with 

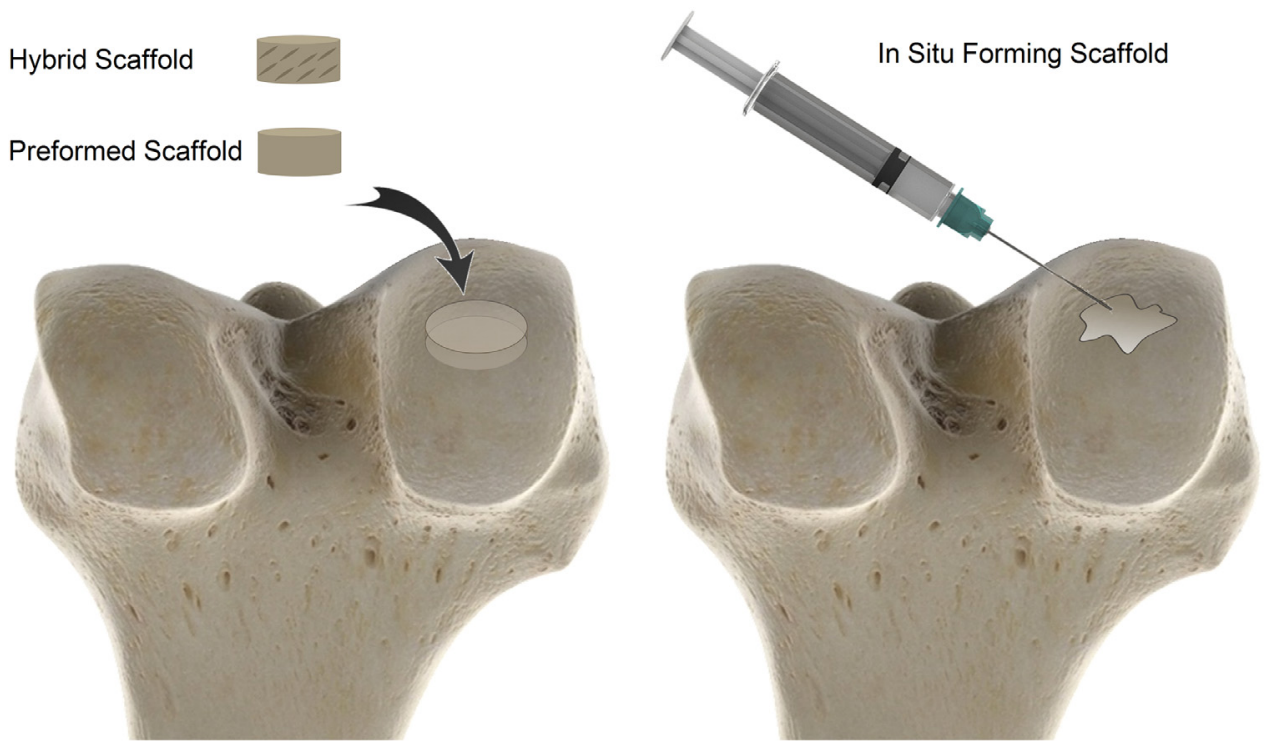

Fig. 2. Schematic illustration of the preformed, hybrid and in situ forming biomaterials.

umbilical cord blood-derived stem cells has also shown enhanced and stable clinical outcomes during a 7-year follow-up study in Korea [45]. Spherox presents another elegant engineered product for the treatment of cartilage injuries. Consisted of chondrocyte aggregates, autologous spheroids are inserted arthroscopically in the injured site where they start healing the defect. The efficacy of Spherox has been shown in two clinical trials (one Phase II and one Phase III) where statistically significant improvement of clinical scores was seen in both studies[46].

\section{Biomaterial platforms: considerations and state-of-the-art}

The overarching principle for effectively choosing the desired material in any tissue engineering applications is having meticulously defined design criteria, that are determined, predominantly, by the final use [47]. More precisely, in case of cartilage regeneration, biomaterial's design, composition and interactions with cells need to be screened and subsequently enhanced based on the interactions with the native cartilage tissue, its inherent repair mechanisms, as well as its ability to be fabricated at a clinically relevant scale. Crucial prerequisites of any biomaterial to be used, present its biocompatibility and degradation properties. The threedimensional biomaterial must be able to support and enhance cell growth and maintenance, while its degradation rate should match the defect healing rate [48].

Additionally, in case of cartilage regeneration, the ideal biomaterial should also allow the recreation of tissue's composition by means of the ratio between the liquid and solid phase of the soft tissue, it should reproduce its zonal architecture, and facilitate a suitable environment for the successful and efficacious integration of the newly-developed tissue with the adjoining native one [49]. Functional regeneration can only be reached via distinctive hierarchical structures, suitable cells that will initiate matrix deposition and a well-orchestrated interplay of mechanical properties. This latter concept, has initiated a quest among the scientific community for the most effective way to substitute for damaged cartilage and to produce the optimal cartilage replacement material.

Biomimetic scaffolds with tissue-like architectures and ondemand properties have long been developed to guide specific cellular responses. For the purpose of this section biomaterials are classified into 3 different categories: Preformed scaffolds, consisting of either naturally derived materials (mainly hydrogels) or syn- thetic materials, in situ forming biomaterials and hybrid biomaterials. Fig. 2 illustrates the differences between these categories.

There is a huge array of preformed biomaterials that have been attempted to regenerate cartilage, spanning from natural-based polymers [50], owning to their extreme similarity with the natural extracellular matrix, to synthetic polymers with various physical and chemical characteristics [51]. Among the various natural biomaterials, chitosan, fibrin, alginate, silk and collagen type I have been extensively investigated for cartilage regeneration. At this point it should be noted that the aforementioned materials can be included under the same title: hydrogels. Hydrogels are three-dimensional hydrophilic networks of cross-linked polymers, which are able to encapsulate large amounts of water [52]. In this regard, hydrogels are usually used to develop a fully hydrated environment for the cells, analogous to the extracellular matrix of the tissue of origin [14].

Alginate for instance, a polysaccharide derived from algae, has been shown to lead to enhanced chondrogenic gene expression levels as well as to elevated production of collagen type II, the main structural component of cartilage tissue. This has been attributed to the fact that chondrocytes can maintain their native round morphology after encapsulation in alginate hydrogels [53]. Collagen type I presents another very common material that has been attempted for cartilage repair. Such scaffolds have facilitated chondrogenesis in studies exploring the effects of dynamic mechanical loading [54], as well as proteoglycans [55, 56]. Silkbased scaffolds have also shown promising results for chondrogenesis when seeded with stem cells. Indeed, silk hydrogels result in improved type II collagen content and proteoglycan synthesis, in seeded mesenchymal stem cells [57]. Fibrin glue and chitosan have also been trialed as potential constructs for cartilage repair due to their excellent biocompatibility and enhanced interactions with chondrocytes [58, 59].

A wide variety of scaffolds for cartilage tissue engineering have also been fabricated by synthetic materials. Unlike naturallyinspired materials, synthetic polymers can be tuned with respect to their physicochemical characteristics [60]. Crucial properties of a polymer such as its mechanical profile or degradation properties can be tuned via alterations in its chemical backbone [61]. Such elegant flexibility inspired tissue engineers to design constructs with predefined biological and mechanical properties. Among the various synthetic polymers, polylactides, polyglycolides and their 
copolymers have been extensively studied for cartilage regeneration [62]. These materials can be customized to form porous constructs, films or meshes, thus allowing for various possibilities for scaffold shape and architecture.

More recently, in situ forming hydrogels were viewed as tempting candidates, owing to their capability of being injected into the body. The attractiveness of such approach stems from the fact of minimally invasive surgery and improved patient's compliance [63]. With this regard, smart, or in other words, stimuli responsive hydrogels have also attracted particular interest. Such hydrogels can undergo various transitions in response to different physicochemical cues, such as changes in $\mathrm{pH}$, temperature, chemical factors or even wavelength or intensity of light. Ehrbar and associates for instance, have developed a novel PEG-based gel that was able to selectively enhance cell adhesion and enzymatic degradation, illustrating the capability of such gels to demonstrate various biomolecular signals [64]. Thermoreversible hydrogels have also been used for cartilage engineering applications. Moghadam et al. (2014) have created a novel poly(2-hydroxyethyl methacrylate) matrix, combined with thermosensitive poly( $\mathrm{N}$-isopropyl acrylamide) nanoparticles. By using its dissipative capabilities as an internal heat source, the scaffold undergoes changes, which result in spatiotemporally control the release of a drug [65].

However, in many cases, despite the fact that hydrogels exhibit unique biocompatibility, their mechanical properties are far lower than those of natural cartilage [66]. To this end, it was thought that the incorporation synthetic or natural fibers in the hydrogel formulation, not only will it enhance the mechanical performance of the hydrogel, but it will also lead to constructs that better resemble cartilage architecture, since cartilage by nature is a composite material. With regards to this latter point, Moutos, Freed and Guilak (2007) generated three-dimensional anisotropic woven constructs, consolidated with a cell-hydrogel blend, demonstrating mechanical performance similar to native's cartilage, as quantified in terms of compressive, tensile and shear testing. The same study also represents proof of concept that cartilage-mimicking constructs, by means of highly controllable mechanical properties and enhanced biological performance could be engineered [67]. In a similar concept, by applying melt electrospinning writing, Bas and associates (2015), reinforced soft hydrogels with well-oriented nanofibrous networks, transforming the latter into hybrid composites with outstanding mechanical performance and enhanced structural integrity [68].

Taken together, the development of functional biomaterials for cartilage restoration presents a highly active area of research, mainly because of the complex physiological characteristics of cartilage tissue. However, despite the promising results that some studies have demonstrated towards cartilage repair through hydrogel-based strategies, clinical success remains limited. From the standpoint of polymer biomaterials, optimization of the mechanical properties and the functionality of the resultant formulation is still a challenge for polymer scientists and engineers. Ideal biomaterials should meet several requirements and should possess various characteristics (as described in Section 2), which demonstrate an active role in cartilage function. In situ forming hydrogels provide tremendous advantages when compared to preformed scaffolds. Unique flexibility, as well as targeted minimal invasive delivery present the most important ones; however, they may also demonstrate a few drawbacks, such as incorporation of toxic crosslinking molecules during the preparation process or very slow rate of gelation at the injured site.

\section{Cell sources}

Even though primary articular chondrocytes seem to be the most obvious choice for regenerating hyaline cartilage, acquiring sufficient number of autologous cells still present a crucial restricting factor for the translation of these cells to the clinic [69]. Sufficient numbers could be possibly obtained by cell expansion in vitro, however this may lead to rapid loss of their chondrogenic phenotype [70]. Addition of growth factors to overcome this issue in vitro is somewhat possible. Chondrocytes expanded for up to passage 3 under the application of transforming growth factor beta 1 during expansion for instance, have been shown to significantly maintained the potential for autonomous cartilage-like tissue formation over the unstimulated control groups [71].

Non-articular chondrocytes such as costal or nasal chondrocytes are also under investigation as alternative cell sources [72, 73]. Similar to articular cartilage, the extracellular environment of costal cartilage is also rich with high amounts of collagen and glycosaminoglycans. In fact, neocartilage produced by costal chondrocytes exhibited mechanical and biochemical properties akin to those of native articular cartilage [73]. Furthermore, chondrocytes obtained from the nasal septum possess higher capacity to produce hyaline-like cartilage tissues and they also exhibit the plasticity to adapt to a "loaded" environment. A nasal-chondrocyte based product is already in clinical trials for the repair of cartilage injuries in Europe [74].

Stem cells have also been deemed as an elegant alternative option. The rationale in using these cells stems from the fact that they can be easily isolated, acquired and expanded in vitro to the desired number and then differentiate to chondrocytes. Regarding cartilage tissue, a lot of research has been focused on mesenchymal stem cells [75, 76], embryonic stem cells [77-79] and more recently to progenitor cells [80]. One problem that arises however, is that hypertrophy may occur during the chondrogenic differentiation of stem cells. Despite promising data, it seems that their long-term efficacy should be further investigated [81].

\section{Mechanobiology in cartilage tissue engineering}

Arguably one of the biggest challenges in cartilage tissue engineering, involves the development of tissue substitutes that not only will they restore the structural features of the tissue, but also its biochemical functions [82]. To accomplish such multifaceted task, the establishment of precise design criteria, in concordance with new fabrication strategies that will aid to develop biomimetic platforms to recapitulate the tissue environment and mimic the cell-environment interactions, present the most important requirements. These design considerations should be relied on a comprehensive understanding of the cellular, biochemical or even on biophysical basis of tissue regulation [83]. In this regard, the central role that the mechanical stresses for instance have on specific cellular responses (e.g. phenotype, growth, differentiation, apoptosis, etc.), especially during the developmental phase, should be taken into consideration. This latter insight is extremely important, since the mechanical stimuli significantly affects cartilage development and function [84].

The pioneering strategy of tissue engineering, primarily focuses on diverse facets of engineering, medicine, biology and biomaterials, but has also expanded to incorporate a broad array of scientific principles, critical for addressing current challenges in the field [85]. Indeed, developing scalable tissue-engineered analogues that will mimic both the architecture and the natural function of native tissues such as cartilage, presents one of the most challenging tasks; however, progress in engineering approaches, more precisely the administration of biophysical cues, have led to the creation of more biomimetic constructs [86,87].

Motivated by the prominent role of the physical and biomechanical interplay between cells and their environment in tissue development and maintenance, mechanobiology has inspired the integration of different types of stimuli into current tissue engi- 
neering approaches [88]. For instance, several studies have demonstrated the beneficial effects of uniaxial compression on chondrogenesis, not only in gene expression levels $[89,90]$ but also in matrix deposition in the 3D constructs [91]. From a mechanical point of view, dynamic compressive loading has also been shown to enhance the mechanical performance of the final construct, by increasing its compressive modulus [92].

Incorporation of such mechanical cues, gives the opportunity to explore the relevance of mechanobiology in functional tissue engineering. More precisely, tissue engineers can leverage specific mechanobiological responses to identify and probe the importance of biomechanical factors in tissue maintenance, control tissue maturation and tune the biosynthetic capacity of the tissue-engineered constructs.

\section{Trends in the 21st century}

With the rapid advancements in the field of tissue engineering, cartilage repair via transplantation of artificial constructs that intimately resemble the characteristics of the natural tissue, has become an attractive alternative option for treating cartilage malfunctions [93]. The major hurdle towards a clinical application of engineered cartilage, centers around the development of functional, yet scalable cartilage constructs, in a credible and reproducible fashion [94]. In this context, numerous research groups have attempted to produce hyaline cartilage constructs in vitro, with some of them demonstrating impressive outcomes [95,12], that may have the potential to be clinically approved and eventually used for the treatment of large cartilage lesions. Identifying the relationship between the cell-mechanical stimuli and developing effective technologies that will aid the fabrication of functional tissue constructs, seem to be the main current focus of the scientific community.

\section{Stimulation using bioreactor systems: biophysical and biochemical} stimuli

Hyaline cartilage can be characterized as a biomechanical tissue. It can respond to various mechanical factors and even though the exact mechanisms involved in the transduction of such forces are not completely elucidated, research highlights the beneficial impact of specific types of stimulation for cartilage synthesis and remodeling. Compressive loading for instance, presents a crucial component of normal stimulation inside the knee joint. Studies have utilized both static and dynamic compression to generate cartilage in vitro [96,97].

Using a custom-made apparatus, Lee and Bader for instance, showed that glycosaminoglycan levels were enhanced, under 15\% compression at $1 \mathrm{~Hz}$ frequency for chondrocytes encapsulated in agarose scaffolds [98]. In another elegant study, Mauck and colleagues observed augmented collagen synthesis and increased equilibrium modulus of cell-seeded scaffolds under compressive loading, compared to free-swelling constructs [99]. Lee et al. also observed an increase of proteoglycan synthesis of chondrocytes when subjected to dynamic compressive loading, while static stimuli resulted in lower matrix deposition [100]. These results, are in agreement with Wernike and associates, who also explored the effects of long-term dynamic compression stimuli on cartilageengineered constructs. Apart from controlling the chondrogenic phenotype, they also achieved high GAG deposition and increased collagen type II expression in the engineered-constructs [101].

Chondrocytes are also subjected to hydrostatic pressure following compressive stimulation of cartilage. More specifically, the low permeability of the tissue does not allow synovial fluid to run out of the tissue and inside the joint area. As a result, pressure inside cartilage cyclically augments during every instance of com- pressive loading [102]. This latter has inspired tissue engineers to utilize hydrostatic pressure to produce cartilage in vitro. In fact, hydrostatic pressure at a physiological range of 5-15 MPa, has been shown to enhance chondrocyte matrix deposition, via modulation of specific chondrocyte transporters [103]. Indeed, a significant improvement in proteoglycan (Aggrecan) production was observed on monolayer chondrocytes, following a $10 \mathrm{MPa}$ static pressure for a period of four hours [103, 104]. Studies utilizing constant pressure have reached physiological levels of biomechanical and biochemical properties in cartilage-engineered substitutes. When bovine chondrocytes experienced $10 \mathrm{MPa}$ of pressure for 1 hour per day for a total of 8 weeks, resulted in enhanced collagen content compared to control groups and prevented the lower glycosaminoglycan content per construct observed in the control groups over time [105]. According to a 2021 study, transient receptor potential channels can mechanotransduce hydrostatic pressure and stimulate matrix formation [102].

Improvements have also been shown when the culturing conditions of the engineered constructs include shear [106]. Shear loading provides a form of stimulation during knee function. The rationale behind shear bioreactor systems that replicate this type of loading usually, center around the idea of producing constructs with enhanced frictional properties more similar to cartilage. As in the case of compressive stimulation or hydrostatic pressure, dynamic shear seems to yield more promising results. Dynamic shear of around $2 \%$ could stimulate $40 \%$ higher collagen synthesis as well as 35\% more proteoglycan production over the control groups [107]. This behavior could be attributed to the activation of primary cilia, a nanoantenna present in chondrocyte channelome. Polycystin I and II, two genes encoding the primary cilia, have been shown to be significantly upregulated when chondrocytes are subjected to shear [108].

At this point it should be mentioned that various types of shear bioreactors exist and have been explored for cartilage tissue engineering. There are systems [109], which try to mimic the natural situation where tissue touches against tissue, there are fluidshear bioreactors that utilize fluid flow as the main source of shear [110] and perfusion bioreactors that have been developed to facilitate nutrient transfer in the engineered constructs [111]. Perfusion systems provide a type of stimulus that is less related to the normal condition of joint movement, however it has been shown that they can also positively influence critical cellular functions. Application of shear force on chondrocyte monolayers demonstrated a 2 -fold increase in glycosaminoglycan production. The same study also showed that the size of the newly-formed glycosaminoglycans was also enhanced in human chondrocytes in response to shear loading [112].

Oxygen concentration presents another potent regulator for cartilage homeostasis. The oxygen concentration of cartilage tissue is estimated in the range of $7-10 \%$ around the superficial zone of the tissue and approximately $1 \%$ close to the subchondral bone. Primary human chondrocytes acquired from healthy [113] and diseased [114] regions of articular cartilage showed increased transcript levels of critical chondrogenic markers under low oxygen conditions. Transforming growth factor signaling pathways, which in turn regulate cartilage development and maintenance, have also been shown to be enhanced when culturing chondrocytes in hypoxia conditions. In fact, a $2.5 \%$ of oxygen concentration is considered optimal for boosting the transcription of such factors [115]. Studies have also utilized hypoxia conditions in synergy with mechanical loading. For example, the combination of dynamic compression and hypoxia led to a significant down-regulation of collagen type I expression, resulting in stabilization of the chondrogenic phenotype [116]. In this regard, hypoxia inducible factor 1 (HIF-1) has been regarded as a crucial element for hypoxic induction of chondrogenesis [117]. 

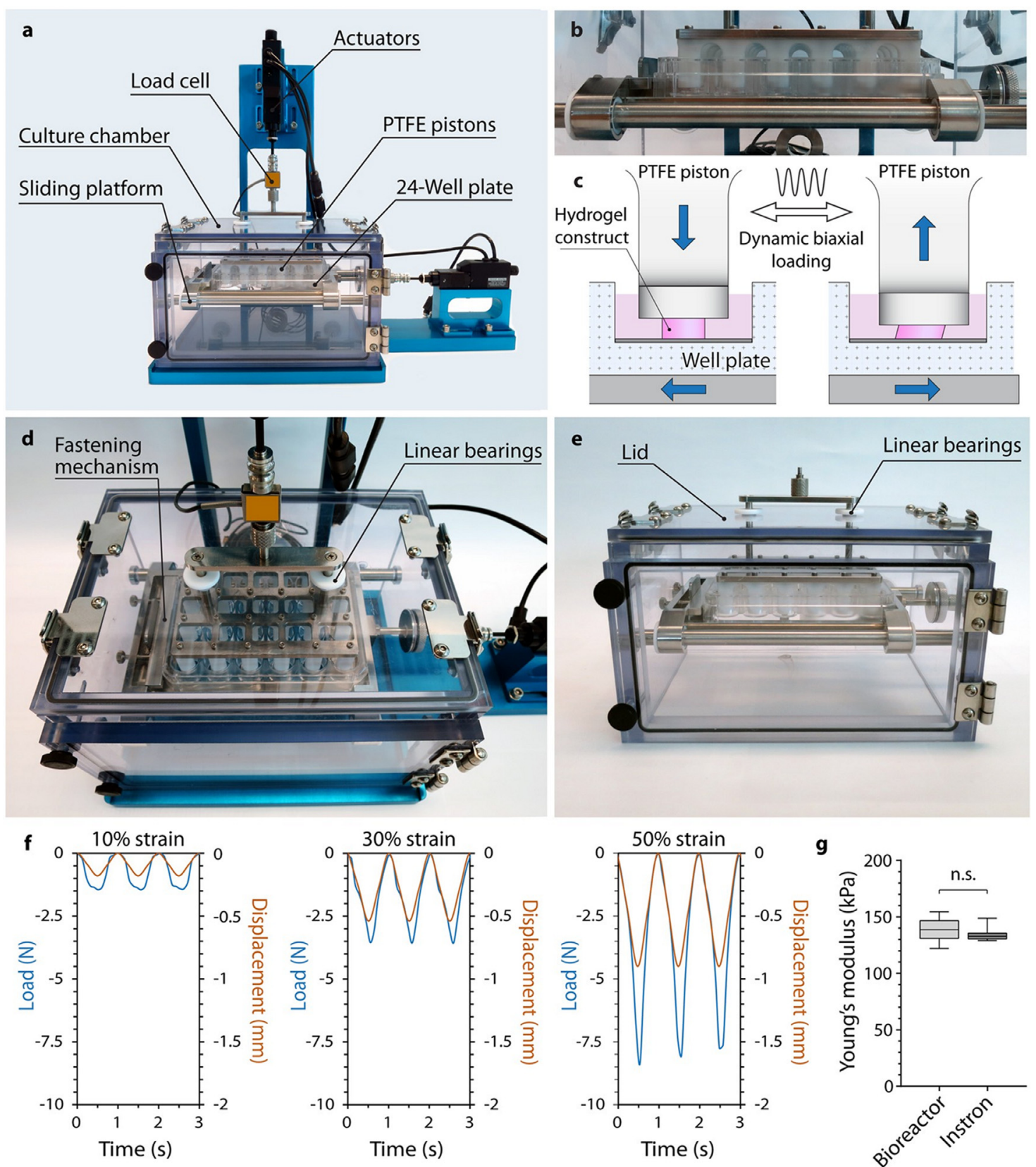

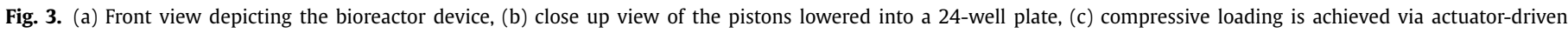

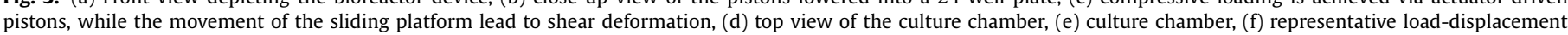
curves, (g) Young's modulus measurement immediately through the bioreactor. Reproduced from [68].

As the field of cartilage engineering expands and matures, more sophisticated bioreactor systems have been developed. Researchers have started integrating various mechanical stimuli into single, hybrid bioreactors in order to elicit a more favorable response from cells. Meinert and colleagues, developed a pioneering bioreactor system that could apply defined uni- or bi-axial mechanical stimulation to the engineered cartilage constructs (Details can be seen in Fig. 3). The resultant system was used to assess the effect of various individual physical cues on chondrocyte mechanobiology. In fact, administration of intermittent biaxial stimulation of cartilageengineered constructs, incorporating clinically relevant cells and biomaterials, was shown to augment matrix accumulation, resulting in cartilage-constructs with superior, natural-like biomechanical properties [118].
Last but not least, tension has also been trialed as an alternative, yet effective stimuli to augment the overall biomechanical performances of neotissue. More specifically, tension stimulation of scaffold-free cartilage-like substitutes, treated with lysyl hydroxylase homolog 2 resulted in an approximate 6-fold increase in tensile strength. Subsequent implantation, illustrated that such constructs exhibited $90 \%$ of the collagen content of native cartilage [119]. The same study showed that transient receptor potential vallinoid 4 ion channel is responsible for transducing tension stimulation. Although the application of tension stimuli was achieved without the use of a bioreactor system, this study impressively illustrates the effectiveness of such biophysical stimuli for the development of functional tissue constructs.

The use of biochemical stimulation in three dimensional cultures have also been explored to enhance the proliferation rate and 
to restore the chondrogenic capacity of articular chondrocytes. Application of insulin-like growth factor 1 (IGF-1) concomitantly with osteogenic protein 1 (OP-1) has been shown to improve matrix secretion in both osteoarthritic and normal chondrocytes, when seeded in alginate [120]. Additionally, culturing primary human chondrocytes with transforming growth factor 2 (TGF- $\beta 2$ ) and insulin growth factor notably improved expression of type II collagen, when compared to control groups [121]. A synergy between transforming growth factor 1 (TGF- $\beta 1$ ) and fibroblast growth factor 2 (FGF-2) has also been suggested. More specifically, when adipose mesenchymal stem cells were treated with the aforementioned factors, a significant up-regulation of cartilage-related genes was observed [122]. It is thought that such factors stimulate critical metabolic programs in chondrocytes, leading to improved matrix synthesis.

Taken together, the potential of bioreactors in improving the quality and efficiency of cartilage engineered constructs has been realised for quite some time. By providing a compendious level of control over a wide range of biophysical and biochemical factors, bioreactors offer the technological means to conduct studies, aiming at revealing which mechanical cue regulates which biological function [123]. Such data will provide crucial information in engineering cartilage tissue. This vital, mechanobiology-related research will offer the basis for tailoring the essential environmental conditions necessary for the generation of functional cartilage tissue.

\section{Cartilage biofabrication}

The emergence of Tissue Engineering was fostered by the augmenting need for functional tissue replacement [124]. The classical paradigm of Tissue Engineering revolves around the use of a three-dimensional carrier, together with cells and other biological factors to regenerate damaged tissues [125]. Despite considerable outcomes in vitro, the lack of engineered substitutes that mimic both the biomechanical and biochemical environment of the native tissue, has hindered its translation into clinical practice [126]. Biofabrication, an emerging research field, encompasses the principles of additive manufacturing and three-dimensional bio-assembly and aims to generate tissue equivalents in an automated fashion, incorporating living cells and biological elements during the process [127]. In other words, it allows for the fabrication of living tissue analogues, via the deposition of living cells and biomaterials onto a substrate from pre-defined computer-generated designs, in a layerby-layer fashion, exclusively and precisely where needed [128].

The ability to produce such precise and complex arrangements might have a beneficial impact to the functionality of the resultant construct, in terms of the accurate and spatial deposition of different types of cells and materials, tailored in such a way, to significantly augment cell response [129]. Reproducing the stratified architecture of native cartilage for instance, that is thought to be one of the main reasons why traditional methods have proven unsuccessful up to now [130], could be easier recapitulated with biofabrication strategies [131]. Indeed, Miguel Castilho and colleagues successfully manufactured biomechanically competent constructs, efficiently mimicking cartilage zonal architecture, that could support neotissue formation upon mechanical stimulation, at physiologically relevant regimes [12].

Furthermore, biofabrication is also believed to demonstrate a crucial role towards improving construct's overall biomechanical performance. From a material standpoint, articular cartilage can be seen as a soft network composite material that plays a crucial role in articulating joints, during dynamic loading [132]. A collagen network together with a proteoglycan-based extracellular matrix, give rise to the viscoelastic nature and stress relaxation of the tissue [133]. However, up to now, the majority of the cartilage- engineered constructs, underperformed during prolonged dynamic loading, suggesting that other alternatives need to be pursued to combat such challenges. In this context, by carefully designing hydrogels that can efficiently emulate the proteoglycan matrix of articular cartilage which were further combined with elegantly designed polymer fibers, Onur Bas and colleagues, managed to fabricate constructs that could capture the overall transient, equilibrium and dynamic mechanical properties of human articular cartilage [82].

As for the feasibility of such strategies to successfully generate clinically relevant constructs, even though there are still hurdles that need to be faced [134], there are, on the other hand, available data illustrating the efficacy of such biofabricated engineered implants. Kang et al. for instance, successfully developed a bioprinting platform and convincingly demonstrated the ability to generate tissue-like structures, such as cartilage, based on CAD data, exhibiting enhanced mechanical integrity and without any sign of necrosis [135]. This specific work successfully exemplified the potential ability of such strategies to overcome current limitations with respect to personalized-shapes and structural integrity.

Ultimately, biofabrication enabled the fabrication of tissue analogues with high geometric complexity and with spatial variations along different axes. The level of control of such techniques offers a valuable tool to better investigate factors that modulate cell responses. Biofabrication of in vitro cartilage substitutes has demonstrated key biomechanical and functional characteristics of the native tissue, and such technology is being embraced as a model system to investigate tissue-level responses.

\section{Preclinical studies: a perspective}

Preclinical studies present an indispensable component in the development of advanced medicinal products and their objective is to illustrate proof of principle of the products as well as define any potential effects that could be predictive for the responses in humans. More specifically, preclinical studies present a central part for tissue engineering applications, since they can provide useful insights regarding the safety and efficiency of biomaterials prior to human clinical trials [137]. Chondrogenesis has been investigated in different animal models, such as nude mice [138,139]. The main advantages of mouse models revolve around the reasonable purchase costs, in concordance with the relatively ease of housing. On the contrary, since the cartilage tissue of mice is far thinner than that of humans, such models might limit the usefulness of the in vivo outcome [140].

Quite a few studies have also utilized rabbits for the assessment of cartilage defect therapy [141]. Rabbits present an attractive alternative, mainly because they are relatively inexpensive, require simple husbandry and their joints are bigger compared to mice or rats [142]. Lin and colleagues (2015), have successfully fabricated composite PLGA/Hap/Zein scaffolds aiming to repair bone-cartilage interface. The subsequent implantation of the composite constructs into rabbits produced invaluable evidence that such scaffolds could induce cartilage formation [143]. On the other hand, it has been shown that rabbits exhibit greater healing capacity compared to bigger animals and humans, a factor that should also be considered before using them in tissue engineering studies [135].

Mini-pig and goat models have also been used in cartilage engineering applications. A thicker cartilage of approximately $1.5 \mathrm{~mm}$ presents a relatively good approximation to that of a human, allowing the study of both partial and full thickness defects. Again, it should be mentioned that it is important to use mature pigs in order to avoid the spontaneous cartilage repair, which may create challenges regarding extrapolating the results. In the case of goats, a high variability in cartilage thickness has been observed, which could lead to fluctuations of the volume of cartilage defects within 
studies and potentially to arbitrary comparisons among studies [132].

Horses are the largest animals that have been used as models in cartilage engineering studies. Since equine joints share several characteristics with those of humans, equine models have been now incorporated for orthopedic research [144]. As an athletic animal, the horse presents an ideal model for the evaluation of strategies in the resurfacing of chronic defects. The cartilage thickness of the horse ranges from 1.75 to $2 \mathrm{~mm}$ for the medial femoral condyle, making it the best candidate for cartilage engineering research [145]. Concerns however center around the loading environment in the horse joint. Although the horse provides a model where defect geometry can match up to the human clinical scenario, its weight (400-500 kg) places lesions under stringent static loading conditions [146].

Cell-based medicinal products, such as chondrocytes containing products can be complex in their development and therefore proof-of-principle preclinical studies are strongly required. In this context, it should be noted that variations between species can present a critical obstacle towards translation to the clinic, since human cells will very likely be rejected by an immunocompetent animal, resulting in a premature elimination of the administered product. Immuno-compromised animals could in this case provide an alternative option, however, dissimilarities in the in vivo environment may also lead to differences in the product-tissue interactions. In such cases, homologous animal models utilizing the respective cells from the same animal species are preferred. The nature and characteristics of the homologous product, along with the manufacturing process must be representative of the product to be used in humans [147-149].

Overall, the selection of appropriate animal models presents one of the most challenging steps for the design of preclinical studies. This is because even basic characteristics, critical to reproduce the situation in patients, may be absent or different in the selected model. The benefit of the different animal models for safety testing must be critically considered and their relevance should be scientifically justified. The selected animal model should replicate the clinical condition of the patient as precise as possible with same pathophysiology as in patients. Normally small animal models are widely employed, in the case of chondrocyte-based products, due to their relative ease of manipulation and due to the fact that are readily available [147]. On the contrary, sometimes data extrapolation from small animal models to humans may be challenging. In this case, larger animal models may be required. In any case, the total number of animals per individual study should allow for statistically and biologically significant interpretation of the results [148]. All safety issues emerging from the non-clinical studies should be carefully addressed, particularly in the presence of physiologic differences that would potentially limit the predictive value of the homologous animal model. Certain notice should be given to biological processes that could potentially stimulate infections or change of cell native characteristics during development and post marketing phase of cartilage products [149].

\section{Requirements for clinical translation}

Tissue engineering has been considered as the new panacea in tissue reconstruction since the last three decades [150]. The term tissue engineering refers to a multidisciplinary strategy that aims to regenerate natural tissues that would last the patient's lifetime [151]. Based on this latter point, a colossal amount of effort and resources have been afforded on tissue engineering research. However, as Scott J Hollister have said, despite almost 30 years of research expenditure in tissue engineering, only a handful of new repair strategies ever attain regulatory approval for clinical use [152].
This number is further decreased, when we consider products that are meant to be used only for cartilage repair.

Therefore, despite some already proposed legislation, the question that immediately arises centers around the "gap" existing between cartilage tissue engineering research and commercialization. This paper asserts that such a gap can be mainly ascribed to regulatory prerequisites, but it can be potentially bridged by focusing on 4 key steps:

1 Manufacturing processes: manufacturing processes should follow the quality system regulations (QSR), so that can potentially obtain FDA or EMA approval.

2 Bioreactor designs \& Mechanobiology: Bioreactor platforms can effectively recapitulate the in vivo environment, and thus can arouse a highly efficacious chondrogenesis. They offer the possibility to assess pioneering salubrious strategies, while minimizing ethical concerns.

3 Pre-clinical experiments: first stage evaluation of the tissueconstruct that has been made in step 1. A more comprehensive understanding regarding the efficiency of the engineered construct can be achieved.

4 Early/first-step clinical trials: based on the outcome of step 3, early stage trials should be performed.

Arguably regulatory prerequisites present the most important factor when considering the successful commercialization of any product that is meant to be used in bio-applications. In cartilage tissue engineering specifically, the resultant tissue-substitutes are usually composed of a biomaterial that act as scaffold, including one or more biological components. It can therefore be understood that based on the exact components of the tissue-analogue, each individual compartment may have to be tested for safety reasons, not only individually, but also in combination with the other components of the "system" [153]. Fig. 5 illustrates the steps towards a potential clinical translation of a cartilage-engineered product.

\section{Manufacturing processes}

Design and manufacturing processes have been considered to hold remarkable promise for the translation of tissue engineering research to a commercialized product, through the fabrication of scalable scaffolds, that will be subsequently used in preclinical studies [154]. Unfortunately, the limiting number of EMA or FDA compliant design and manufacturing facilities hinders the translation of tissue engineering-based strategies for cartilage repair. It is of high importance that such manufacturing sites operate under the appropriate FDA regulations [155]. As it has already being emphasized, depending on the complexity of the tissue-substitute, different regulations may need to be applied for the approval of the final product. For this reason, manufacturing companies need to consult the EMA or FDA on a case-to-case basis. Furthermore, Good Tissue Practice presents an absolute necessity towards the successful transition from the bench to bedside. Good Tissue Practice, is considered as part of the Good Manufacturing Practice, and ensures the generation of a sterile, safe and of high efficiency product. Therefore, such guidelines should be taken into account when considering the commercialization of a product [156].

\section{Bioreactor designs}

Bioreactors represent feasible and effective tools to study the mechanobiology of cartilage tissue, under more physiologically relevant conditions compared to any of the various simplified cell cultures [123]. For tissues of the musculoskeletal system like cartilage, it has been proved by numerous studies that different kinds of mechanical stimulation, seems to favor the tissue in culture $[98,99,102]$. The key motivation behind the use of such systems 
are precisely defined biological and mechanical conditions [157]. Elucidating and characterizing the potential effects of the different biophysical stimuli that the engineered cells may sense in vivo, is proposed to present a cornerstone for bridging the gap between research and clinical translation. Indeed, substantial evidence regarding the long-term capabilities and performance of the engineered constructs can be acquired from such information.

Mechanical stress is viewed as a key regulator of cell response and there is a global agreement that the controlled application of such stimuli, through novel bioreactor designs that mimic the in vivo complex tissue environment, can be utilized to control construct maturation, at a clinically relevant scale, in a precise and reproducible fashion [158]. Since reproducibility and scalability are considered as the gatekeepers for the bench-to-the-bedside jump [154], using cell-seeded scaffolds directed under different mechanical stimuli to generate stable and defined tissue substitutes in vitro, are key endeavours believed to achieve clinically translational success in vivo.

\section{Preclinical testing}

Clinical translation of engineered substitutes for cartilage repair present a challenging endeavor. Such products are considered as "high-risk" devices and for that reason the implementation of predictive animal models present a necessary requirement, prior to a first or even a mid-stage clinical trial. The main purpose of animal models centers around the proof of safety and durability of the product, prior to clinical testing [159]. Although there is no animal model that allows for direct application to humans, each can be used for yielding principles on which conclusions can be reached, that might be beneficial for the eventual translation into a human application. However, the right choice of the appropriate animal model is of significant importance. The safety studies should be designed in such a way to produce clinically meaningful and reliable information to support safe use of the product in the intended clinical application. Therefore, safety studies in nonrelevant species may be misleading.

In a very elegant review article, Reinholtz and colleagues pointed out that the important issue regarding the selection of the right animal model is how close actually the model is to the question being investigated and the hypothesis to be tested [160]. Animals such as goats, sheeps and pigs have all been used as models for cartilage tissue engineering studies, however, meticulous consideration should be given into factors associated with animal maturity, since the metabolic turnover of cartilage tissue in mature individuals is nil. Having said that, Wei and Messner observed that the spontaneous healing of full-thickness osteochondral lesions in the knee joints of immature rabbits showed an accelerated filling and earlier tissue specialization compared to adult animals [161].

Quantitative and qualitative outcome measures should be taken into consideration, since they can provide reliable data to support investment decisions and subsequent applications to regulatory bodies for clinical trials. A wide variety of histological, ultrastructural, biochemical, and biomechanical assessments are available to assess engineered neocartilage. Various types of stains such as hematoxylin/eosin, alcian blue or safranin $\mathrm{O}$ can be used to histologically examine the distribution and position of glycosaminoglycans, collagen type II and other matrix constituents. As for the case of implanted constructs, non-invasive techniques such as ultrasound imaging or magnetic resonance imaging can be utilized.

\section{Clinical trials}

Once the preclinical studies prove the efficacy and the safety of the product, clinical data outcome is required for FDA approval. The clinical trials will be consisted of different phases. An early or phase I trial will focus on the safety of the engineered product and it will involve a limited number of patients. A mid stage or phase II trial will then mainly focus on the efficiency of the corresponding treatment, compared to the most nearly related clinical strategy. However, at this phase a higher number of patients is required to obtain statistical testing. In addition to this latter, for products with expected long-term viability such as in the case of cartilage engineered constructs, patient follow-up is required in order to confirm long term efficacy and safety issue related to the product.

\section{Concluding remarks \& future perspectives}

The multidisciplinary field of cartilage tissue regeneration has undergone exceptional evolution over the past two decades, with radical achievements in various areas as highlighted above. The rapid advancements of new technologies in areas such as biomaterials and biofabrication, coupled with tremendous developments in mechanobiology and bioreactor design, will unquestionably yield a crucial mark on cartilage tissue engineering over the next years. Mechanical signals sensed by chondrocytes profoundly contribute to various anabolic and catabolic responses. Therefore, a more comprehensive understanding of the effects of the different biomechanical stimuli both in native cartilage and in cartilageengineered constructs, in vitro and in vivo, will provide crucial insights that will enhance tissue repair strategies. In this context, a fundamental step in determining the importance of various factors of the mechanical environment, involves the development of novel bioreactor designs that will effectively reproduce the in vivo, yet complex environment of native cartilage. Clinical grade cartilage constructs need to exhibit predictable properties and this can mainly be controlled in bioreactors. Such knowledge will create new horizons towards the establishment of functional protocols that will provide useful guidelines to those who seek to fabricate functional tissue-analogues. However, it is true that a great number of challenges still remain, mainly regarding scaling-up, and manufacturing strategies, that will present important prerequisites prior to getting such technologies transfered to the clinic. Howbeit, the multidisciplinary field of cartilage tissue engineering has come into its own path and we envision that it is very probable to have a quite promising future. Fig. 4

\section{Search strategy and selection criteria}

Information for this review was identified by searches of PubMed, Science Direct and references from relevant articles using the search terms "Cartilage Mechanobiology", "Bioreactors", "Clinical translation of cartilage tissue engineering", and "Biomaterials for cartilage repair". Only articles published in English were included.

\section{References}

[1] Gomoll A.H. and Minas T. The quality of healing: articular cartilage. Wound Repair Regener 2014;22:30-8.

[2] Yuan D., Chen Z., Lin T., Luo X., Dong H., Feng G. Cartilage tissue engineering using combination of chitosan hydrogel and mesenchymal stem cells. J Chem 2015;1:1-6.

[3] Armiento A.R., Alini M. and Stoddart M.J. Articular fibrocartilage-Why does hyaline cartilage fail to repair? Adv Drug Deliv Rev 2019;146:289-305.

[4] Zhang L., Hu J. and Athanassiou K.A. The role of tissue engineering in articular cartilage repair and regeneration. Crit Rev Biomed Eng 2009;37:1-57.

[5] Sancho-Tello M., Forriol F., Martin de Llano J.J., Antolinos-Trupin C., GomezTejedor J.A., Gomez Ribelles J.L. and Carda C. Biostable scaffolds of polyacrylate polymers implanted in the articular cartilage induce hyaline-like cartilage regeneration in rabbits. Int J Artif Organs 2017;40:350-7.

[6] Tsai-Sheng F., Yu-Hong W., Po-Yuan C., I-Ming C. and Wei-Chuan C A. Novel Biodegradable and thermosensitive poly(ester-amide) hydrogel for cartilage tissue engineering. Biomed Res Int 2018;2018:1-12.

[7] Karami P, Nasrollahzadeh N, Wyss C, O'Sullivan A, Broome M, Procter P, et al. An Intrinsically-Adhesive Family of Injectable and Photo-Curable Hydro- 
Design:

Femoral condyle curvature (human knee joint)

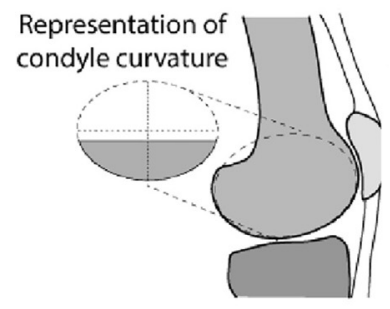

Microfibre patterning with melt electrowriting (MEW)

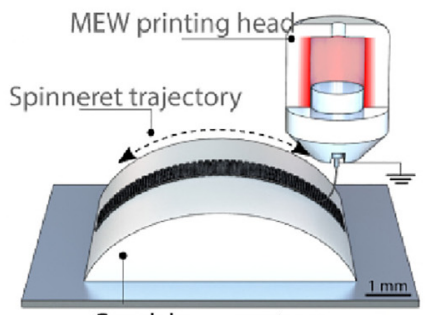

Condyle - curvature of clinically relevant material

Proof of concept

3D printed condyle-curvature resurfaced with reinforcing microfibres

Fig. 4. Melt electrowriting onto anatomically relevant biodegradable substrates: Resurfacing a diarthrodial joint. Reproduced from [136].
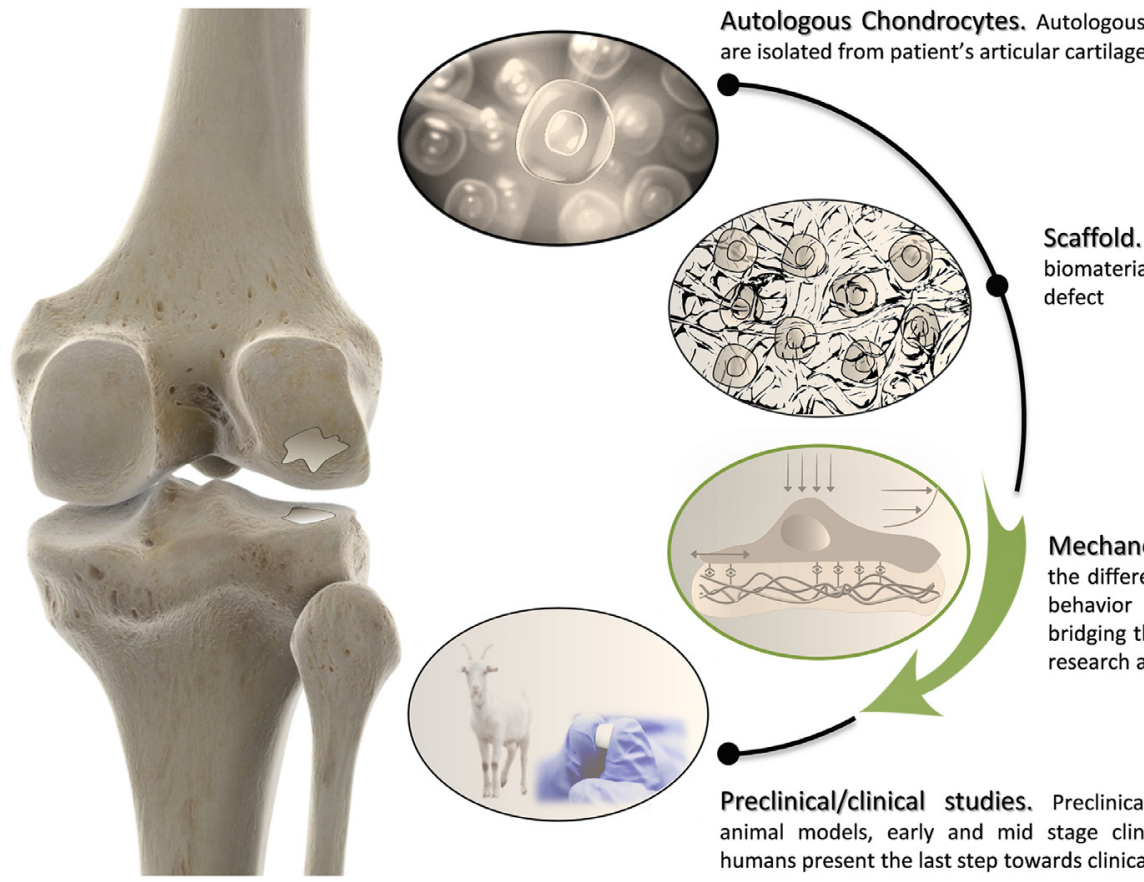

$0^{2}$
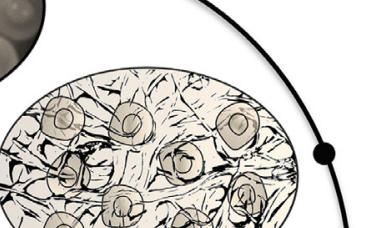

Scaffold. Chondrocytes are seeded in a porous biomaterial, molded into the shape of the defect

Reinforcing fibres

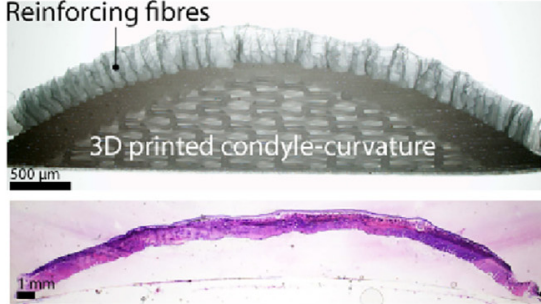

H\&E staining (after 28 days in vitro culture)

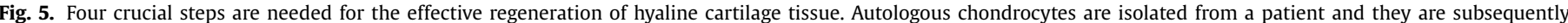

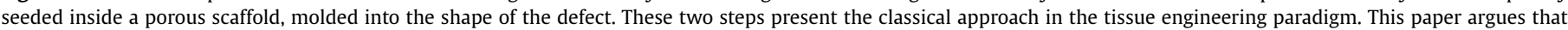

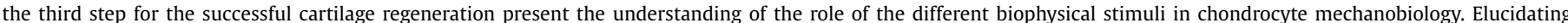

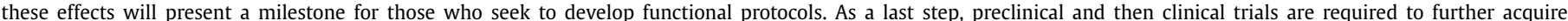
data that illustrate the efficiency of the implanted construct.

gels with Functional Physicochemical Performance for Regenerative Medicine. Macromol Rapid Commun 2021;42(10):2000660.

[8] Nasrollahzadeh N., Karami P. and Pioletti D. Control of dissipation sources: a central aspect for enhancing the mechanical and mechanobiological performances of hydrogels. ACS Appl Mater Interfaces 2019;11:39662-71.

[9] Karami P., Samira Wyss C., Khoushabi A., Schmocker A., Broome M., Moser C., Bourban P.E. and Pioletti D. Composite double-network hydrogels to improve adhesion on biological surfaces. ACS Appl Mater Interfaces 2018;10:38692-9.

[10] Schulz R.M. and Bader A. Cartilage tissue engineering and bioreactor systems for the cultivation and stimulation of chondrocytes. Eur Biophys J 2007;36:539-68.

[11] Schröder C., Andreas H., Ge Z., Matthias W. A closed loop perfusion bioreactor for dynamic hydrostatic pressure loading and cartilage tissue engineering. J Mech Med Biol 2016;16:1-16.

[12] Castilho M., Vivian M., Mike C., Keita, I. Bi-layered micro-fibre reinforced hydrogels for articular cartilage regeneration. Acta Biomater 2019;95:297-306.

[13] O'Conor C., Leddy H., Benefield H., Liedtke W. and Guilak F. TRPV4-mediated mechanotransduction regulates the metabolic response of chondrocytes to dynamic loading. PNAS 2014;111:1316-21.

[14] Visser J., Melchels F.P.W., Jeon J.E., Bussel E.M., Kimpton L.S., Byrne H.M., et al. Reinforcement of hydrogels using three-dimensionally printed microfibres. Nat Commun 2015;6:1-10.

[15] Bas O., Lucarotti S., Angella D., Castro N., Meinert C., Wunner F. et al. Rational design and fabrication of multiphasic soft network composites for tissue engineering articular cartilage: a numerical model-based approach. Chem Eng J 2018;340:15-23.

[16] Bas O., Catelas I., De-Juan-Pardo E. and Hutmacher D. The quest for mechanically and biologically functional soft biomaterials via soft network composites. Adv Drug Deliv Rev 2018;132:214-34.

[17] Warnecke D., Maxi M., Luis R., Svenja S., Cristina G., Robert W., et al. Articular cartilage and meniscus reveal higher friction in swing phase than in stance phase under dynamic gait conditions. Sci Rep 2019;9:1-9.

[18] Triche R. and Mandelbaum B.R. Overview of cartilage biology and new trends in cartilage stimulation. Foot Ankle Clin 2013;18:1-12.

[19] Quiroga J.M., Wilson W., Ito K., Donkelaar C.C. Relative contribution of articular cartilage's constitutive components to load support depending on strain rate. Biomech Model Mechanobiol 2017;16:151-8

[20] Hossain M.J., Noori-Dokht H., Sonali K., Alyafei N., Joukar A., Trippel B.S.,et al. Anisotropic properties of articular cartilage in an accelerated in vitro wear test. J Mech Behav Biomed Mater 2020;109:103834.

[21] Han E., Chen S.S., Klisch M.S., Sah L.R. Contribution of proteoglycan osmotic swelling pressure to the compressive properties. Biophys J 2011;101:916-24.

[22] Mow V.C., Ratcliffe A. and Robin Poole A. Cartilage and diarthrodial joints as paradigms for hierarchical materials and structures. Biomaterials 1992;13:90001-5 
[23] Askew M.J. and Mow V.C. The Biomechanical Function of the Collagen Filril infrastructure of Articular Cartilage. J Biomech Eng 1992;100:105-15.

[24] Parra N., Alvarado D., and Carlos J. Mechanical Behavior of Articular Cartilage. In; Goswami T, eds. Injury and skeletal biomechanics; 2012.

[25] Szarko M., and Xia Y. Direct visualisation of the depth-dependent mechanical properties of full-thickness articular cartilage. Open J Orthop 2012;2:34-9.

[26] Antons J., Marascio M.G.M., Nohava J., Martin R., Applegate L.A., Bourban P.E., et al. Zone-dependent mechanical properties of human articular cartilage obtained by indentation measurements. J Mater Sci Mater Med 2018;29:29-57.

[27] Vaishya R. The journey of articular cartilage repair. J Clin Orthop Trauma 2016;7:135-6.

[28] Benedek T.G. A history of the understanding of cartilage. Osteoarthr Cartil 2006;14:203-9.

[29] Hunter W. Of the structure and diseases of articu-lating cartilages. Roy Soc Lond Phil Trans 1743;9;514-21.

[30] Grande D.A., Schwartz A.J., Brandel E., Chahine O.C., Sgaglione N. Articular cartilage repair: where we have been, where we are now, and where we are headed. Cartilage 2013;4: 281-5.

[31] Hunziker E.B., Lippuner K., Keel, M.B., Hintani N. An educational review of cartilage repair: precepts \& practice - myths \& misconceptions - progress \& prospects. Osteoarthr Cartil 2015;23:334-50.

[32] Steadman R, Rodkey W, Briggs K. Microfracture to treat full-thickness chondral defects: surgical technique, rehabilitation and outcomes. J Knee Surg 2002:15:170-6.

[33] Gudas R., Gudaite A., Pocius A., Gudiene A., Cekanauskas E. et al. Ten-Year follow-up of a prospective, randomized clinical study of mosaic osteochondral autologous transplantation versus microfracture for the treatment of osteochondral defects in the knee joint of athletes. Am J Sport Med 2012;40:2499508.

[34] Mirza Z., Swenson R. and Lynch S. Knee cartilage defect: marrow stimulating techniques. Curr Rev Musculoskelet Med 2015;8:451-6.

[35] Bartha L., Vajda A., Duska Z., Rahmeh H. and Hangody L. Autologous osteochondral mosaicplasty grafting. J Orthop Sports Phys Ther 2006;36:739-50.

[36] Haber D., Logan C., Murphy C., Sanchez A., LaPrade R. and Provencher M. Osteochondral Allograft Transplantation for the Knee: post-operative rehabilitation. Int J Sports Phys Ther 2019;3:487-99.

[37] Levy Y., Gortz S., Pulido P., McCauley J. and Bugbee W. Do fresh osteochondral allografts successfully treat femoral condyle lesions? Clin Orthop Relat Res 2013;471:231-7.

[38] Hangody and Fules P. Knee autologous osteochondral mosaicplasty for the treatment of full-thickness defects pf weight-bearing joints. JB\&JS 2003;85:25-32.

[39] Davies R. and Kuiper N. Regenerative Medicine: a Review of the evolution of Autologou Chondrocyte Implantation (ACI) Therapy. Bioengineering 2019;6:116.

[40] Ebert J., Fallon M., Janes G. and Wood D. Prospective clinical and radiologic evaluation of patellofemoral matrix-induced autologous chondrocyte implantation. Am J Sport Med 2017; 6:1362-72.

[41] Gou G.H., Tseng F.J., Wang S.H., Chen P.J., Shyu J.F., Weng C.F., et al. Autologous chondrocyte implantation versus microfracture in the knee: a meta-analysis and systematic review. Arthroscopy 2020;36:289-303.

[42] Musumeci G., Loreto C., Castorina S., Imbesi R., Leonardi R., Castrogiovanni P. Current concepts in the treatment of cartilage damage. A review. IJAE 2013; 118:189-203.

[43] Phase iii study to evaluate safety and effectiveness of novocart 3D plus vs microfracture in knee cartilage defects (N3D). 2012. Available from URL: https: //clinicaltrials.gov/ct2/show/NCT01656902.

[44] McCormick F., Cole B., Nwachukwu B., Harris Joshua, Davis A. and Farr Jack. Treatment of focal cartilage defects with a juvenile allogenic 3-dimensional articular cartilage graft. Oper Tech Sports Med 2013;2:95:93.

[45] Park Y., Ha C., Lee C., Yoon Y. and Park Y. Cartilage regeneration in osteoarthritic patients by a composite of allogeneic umbilical cord bloodderived mesenchymal stem cells and hyaluronate hydrogel: results from a clinical trial for safety and proof-of-concept with 7 years of extended followup. SCTM 2017;6:613-21.

[46] European Medicines Agency. New advanced therapy to repair cartilage defects in the knee. 2017. Available at URL: https://www.ema.europa.eu/en/ news/new-advanced-therapy-repair-cartilage-defects-knee

[47] Shoichet M.S. Polymer scaffolds for biomaterials applications. Macromolecules 2010;43:581-91.

[48] Armiento A.R., Stoddart M.J., Alini M., Eglin D. Biomaterials for articular cartilage tissue engineering: learning from biology. Acta Biomater 2018;65:1-20.

[49] Izadifar Z., Chen X. and Kulyk, W. Strategic design and fabrication of engineered scaffolds for articular cartilage repair. J Funct Biomater 2012;3:799838.

[50] Kim W.K., Choi J.H., Shin M.E., Kim J.W., Kim P., Kim N., et al. Evaluation of cartilage regeneration of chondrocyte encapsulated gellan gum-based hyaluronic acid blended hydrogel. Int J Biol Macromol 2019;141:51-59.

[51] Frassica M. and Grunlan M. Perspectives o synthetic materials to guide tissue regeneration for osteochondral defect repair. ACS Biomater Sci Eng 2020;8:4324-36.

[52] Spicer C.D. Hydrogel scaffolds for tissue engineering: the importance of polymer choice. Polym Chem2020;11:184-219.

[53] Murphy C. and Sambanis A. Effect of oxygen tension and alginate encapsulation on restoration of the differentiated phenotype of passaged chondrocytes. Tissue Eng. 2004;7:1-23.
[54] Hunter C., Imler S., Malaviya P., Nerem R. and Levenston M. Mechanical compression alters gene expression and extracellular matrix synthesis by chondrocytes cultured in collagen I gels. Biomaterials 2002;23:1249-59.

[55] Lee C., Breinan H., Nehrer S. and Spector M. Articular cartilage chondrocytes in type $\mathrm{i}$ and type II collagen-GAG matrices exhibit contractile behavior in vitro. Tissue Eng. 2000;6:555-65.

[56] van Susante J.L.C., Pieper J., Buma P., van Kuppevelt T.H., van Der Kraan P.M et al. Linkage of chondroitin-sulfate to type I collagen scaffolds stimulates the bioactivity of seeded chondrocytes in vitro, Biomaterials, 2001;17:2359-69.

[57] Meinel L., Hofmann S., Karageorgiou V., Zichner L., Langer R., Kaplan D. and Vunjak-Novakovic G. Engineering cartilage-like tissue using human mesenchymal stem cells and silk protein scaffolds. Biotechnol. Bioeng. 2004;8:451-6.

[58] Bobhome-Espinosa A., Campos F., Durand-Herrera D., Sanchez-Lopez J., Schaub S., Duran J. et al. In vitro characterization of a novel magnetic fibrin-agarose hydrogel for cartilage tissue engineering. J Mech Behav Biomed 2020;104:103619.

[59] Shamekhi M., Mirzadeh H., Mahdavi H., Rabiee A., Mohebbi-Kalhori D. and Eslaminejad M. Graphene oxide containing chitosan scaffolds for cartilage tissue engineering. Int J Biol Macromol 2019;127:396-405.

[60] Samira Wyss C., Karami P., Bourban P.E. and Pioletti D. Hybrid granular hydrogels: combining composites and microgels for extended ranges of material properties. Soft Matter 2020;16:3769.

[61] Wei W., Ma Y., Yao X., Zhou W., Wang X., Li C., Lin J. et al. Advanced hydrogels for the repair of cartilage defects and regeneration. Bioact Mater 2021;6:9981011.

[62] Jeuken R., Roth A., Peters R., Donkelaar C., Thies J Rhijn L. and Pieter Emans. Polmers in cartilage defect repair of the knee: current status and future prospects. Polymers (Basel) 2016;8:219.

[63] Fan W., Yuan L., Li J., Wang Z., Chen J., Guo C., et al. Injectable double-crosslinked hydrogels with kartogenin-conjugated polyurethane nanoparticles and transforming growth factor $\beta 3$ for in-situ cartilage regeneration. Mater Sci Eng 2020;110:110705.

[64] Ehrbar M., Rizzi S.C., Schoenmakers R.G., Miguel B.S., Hubbel J.A., Weber F., et al. Biomolecular hydrogels formed and degraded via site-specific enzymatic reactions. Biomacromolecules 2007;8:3000-7.

[65] Moghadam M.N., Kolesov V., Vogel A., Klok H.A., Pioletti D. Controlled release from a mechanically-stimulated thermosensitive self-heating composite hydrogel. Biomaterials 2014;35:450-5.

[66] Beddoes C.M., Whitehouse M.R., Briscoe W.H., Su B. Hydrogels as a replacement material for damaged articular hyaline cartilage. Materials (Basel) 2016;9:1-19.

[67] Moutos F.T., Freed L.E. and Guilak F. A biomimetic three-dimensional woven composite scaffold for functional tissue engineering of cartilage. Nat Mater 2007;6:162-7.

[68] Bas O., Pardo E.M.J., Chhaya, M.P., Wunner F.M., Jeon J.J., Klein T.J., et al. Enhancing structural integrity of hydrogels by using highly organised melt electrospun fibre constructs. Eur Polym 2015;72:451-63.

[69] Roelofs A., Rocke J. and Bari C. Cell-based approaches to joint surface repair: a research perspective. Osteoarthr Cartil 2013;21:892-900.

[70] Medvedeva, E., Grebenik E., Gornostaeva S., Telpuhov V., Lychagin A., Timashev P. and Chagin A. Repair of damaged articular cartilage: current approaches and future directions. Int J Mol Sci 2018;19:2366.

[71] Tekari A., Luginbuehl R., Hofstetter W. and Egli R. Transforming growth factor beta signalling is essential for the autonomous formation of cartilage-like tissue by expanded chondrocytes. PLoS ONE 2015;10:e0120857.

[72] Huwe L., Brown W., Hu J. and Athansiou K. Characterization of costal cartilage and its suitability as a cell source for articular cartilage tissue engineering. J Tissue Eng Regen Med 2018;12:63-1176.

[73] Peltari K., Pippenger B., Mumme M., Feliciano S., Scotti C., Mainil-Varlet P., Procino A. et al. Adult human cres-derived cells for articular cartilage repair. Sci Transl Med 2014;6:251ra119.

[74] Mumme M., Barbero A., Miot S., Wixmerten A., Feliciano S., Wolf F. et al Nasal chondrocyte-based engineered autologous cartilage tissue for repair of articular cartilage defects: an observational first-in-human trial. The Lancet 2016;388:1985-94

[75] Satue M., Schuler C., Ginner N. and Erben R. Intra-articularly injected mesenchymal stem cells promote cartilage regeneration, but do not permanently engraft in distant organs. Sci Rep 2019;9:10173.

[76] Goldberg A., Mitchell K., Soans J., Kim L. and Zaidi R. The use of mesenchymal stem cells for cartilage repair and regeneration: a systematic review. J Orthop Surg Res 2017;12:39.

[77] Nakayama N., Duryea D., Manoukian R., Chow Gand Han C. Macroscopic cartilage formation with embryonic stem-cell-derived mesodermal progenitor cells. J Cell Sci 2003;116:2015-28.

[78] Sui Y., Clarke T. and Khillan J. Limb bud progenitor cells induce differentiation of pluripotent embryonic stem cells into chondrogenic lineage. Differentiation 2003;71:578-85.

[79] Tanaka H., Murphy C., Murphy C., Kimura M., Kawai S. and Polak J. Chondrogenic differentiation of murine embryonic stem cells: effects of culture conditions and dexamethasone. J Cell Biochem 2004;93: 454-62.

[80] Laurent A., Abdel-Sayed P., Ducrot A., Hirt-Burri N., Scaletta C., Jaccoud S., Nuss K., Buys A., Raffoul W., Pioletti D., et al. Development of standardized fetal progenitor cell therapy for cartilage regenerative medicine: industrial transposition and preliminary safety in xenogeneic transplantation.

[81] Somoza R., Welter J., Correa D. and Caplan A. Chondrogenic differentiation of 
mesenchymal stem cells:challenges and Unfulfilled Expectations. Tissue Eng Part B 2014;20:596-608.

[82] Lammi M.J., Piltti J., Prittinen J., Qu C. Challenges in fabrication of tissueengineered cartilage with correct cellular colonization and extracellular matrix assembly. Int J Mol Sci 2018;19:2700-20.

[83] Ingber DE. Mechanobiology, Tissue Development and Organ Engineering in Principles of Tissue Engineering. In: Robert L, Robert L, Joseph V, editors Principles of tissue engineering. Elsevier; 2013. p. 309-22.

[84] Fahy N., Alini M. and Stoddart M.J. Mechanical stimulation of mesenchymal stem cells: implications for cartilage tissue engineering. J Orthop Res 2018;36:52-63.

[85] Guilak F., Butler D.L., Goldstein S.A., Baaijens F.P.T. Biomechanics and mechanobiology in functional tissue engineering. J Biomech. 2015;47:193340.

[86] Salinas E.Y., Hu J.C. and Athanasiou K. A Guide for Using Mechanical Stimulation to Enhance Tissue-Engineered Articular Cartilage Properties. Tissue Eng Part B Rev 2018;24:345-58.

[87] Butler R.J., Melvin N.J., Boivin G.P., Galloway M.T., Shearn J.T., Gooch C., et al. Functional tissue engineering for tendon repair: a multidisciplinary strategy using mesenchymal stem cells, bioscaffolds, and mechanical stimulation. J Orthop Res 2007;25:1121-7.

[88] Kurniawan N.A. The ins and outs of engineering functional tissues and organs. Curr Opin Organ Transplant2019;24:590-7.

[89] Li Z., Kupcsik L., Yao S.J., Alini M., Stoddart, M.J. Mechanical load modulates chondrogenesis of human mesenchymal stem cells through the TGF- $\beta$ pathway. JCMM 2010;14:1338-46.

[90] Mauck R., Byers, B.A., Yuan X., Tuan R.S. Regulation of cartilaginous ECM gene transcription by chondrocytes and MSCs in 3D culture in response to dynamic loading. Biomech Model Mechanobiol 2007;6:113-25.

[91] Nicodemus G.D. and Bryant S.J. Mechanical loading regimes affect the anabolic and catabolic activities by chondrocytes encapsulated in PEG hydrogels. Osteoarthr Cartil 2010;18:126-37.

[92] Appelman T.P., Mizrahi J., Elisseeff J.H., Seliktar D. The differential effect of scaffold composition and architecture on chondrocyte response to mechanical stimulation. Biomaterials 2009;30:518-25.

[93] Chung C. and Burdick J.A. Engineering cartilage tissue. Adv Drug Deliv Rev 2008;60:243-62.

[94] Madry H., Alini M., Stoddart M.J., Evans C., Miclau T., Steiner S. Barriers and strategies for the clinical translation of advanced orthopaedic tissue engineering protocols. Eur Cells Mater 2014;27:17-21.

[95] Bas O., Pardo E.M.J., Meinert C., Angella D., Baldwin J.G., Bray L.J., et al. Biofabricated soft network composites for cartilage tissue engineering. Biofabrication 2017;9:25014

[96] Palmoski M. and Brandt K. Effects of static and dynamic loading on articular plugs in vitro. Arthritis Reumatol 1984;27:675-81.

[97] Schilz R., Wustneck N., van Donkelaar C., Shelton J. and Bader A. Development and validation of a novel bioreactor system for load- and perfusioncontrolled tissue engineering of chondrocyte-constructs. Biotech and Bioeng 2008;101:714-28.

[98] Lee DA and Bader D.L. Compressive strains at physiological frequencies influence the metabolism of chondrocytes seeded in agarose. J Orthop Res 1997;15: 181-8.

[99] Mauck R.L., Soltz M.A., Wang C.C., Wong D.D., Chao P.H., Valhmu W.B., et al. Functional tissue engineering of articular cartilage through dynamic loading of chondrocyte-seeded agarose gels. J Biomech Eng 2000;122:252-60.

[100] Lee C.R., Grodzinsky A.J. and Spector M. Biosynthetic response of passaged chondrocytes in a type II collagen scaffold to mechanical compression. J Biomed Mater Res A 2003;64:560-9.

[101] Wernike E., Li Z., Alini M., Grad S. Effect of reduced oxygen tension and longterm mechanical stimulation on chondrocyte-polymer constructs. Cell Tissue Res 2008;331:473-83.

[102] Savadipour A., Nims R., Katz D. and Guilak F. Regulation of chondrocyte biosynthetic activity by dynamic hydrostatic pressure: the role of TRP channels. Connect Tissue Res 2021:1-13.

[103] Hall A.C. Differential effects of hydrostatic pressure on cation transport pathways of isolated articular chondrocytes. J. Cell. Physiol 1999;178:197-204.

[104] Smith L., Rusk S., Ellison B., Wessells P., Tsuchiya K., Carter D. et al. In vitro stimulation of articular chondrocyte mRNAand extracellular matrix synthesis by hydrostatic pressure. Orthop Res 1996;14:53-60.

[105] Elder B. and Athanasiou K. Hydrostatic Pressure in Articular Cartilage Tissue Engineering:from Chondrocytes to Tissue Regeneration. Tissue Eng 2009;15:43-53.

[106] Smith R.L., Lin J., Trindade M.C., Shida J., Kajiyama G., Vu T., et al. Timedependent effects of intermittent hydrostatic pressure on articular chondrocyte type II collagen and aggrecan mRNA expression. JRRD 2000;37:153-61.

[107] Waldman S., Spiteri C., Grypnas M., Pilliar R. and Kandel R. Long-term intermittent shear deformation improves the quality of cartilaginous tissue formed in vitro. Orthop Res 2006;21: 590-6.

[108] Salinas E., Aryaei A., Paschos N., Berson E., Kwon H., Hu J. and Athanasiou K. Shear stress induced by fluid flow produces improvements in tissueengineered cartilage. Biofabrication 2020;12: 045010.

[109] Frank E., Jin M., Loening A., Levenston M. and Grodzinsky A. A versatile shear and compression apparatus for mechanical stimulation of tissue culture explants. J Biomech 2000;33:1523-7.

[110] Bueno E., Bilgen B. and Barabino G. Wavy-walled bioreactor supports in- creased cell proliferation and matrix deposition in engineered cartilage constructs. J Tissue Eng 2006;11:11-12.

[111] Mizuno S Alleman F. and Glowacki J. Effects of medium perfusion on matrix production by bovine chondrocytes in three-dimensional collagen sponges. J Biomed Mat Res 2001;56: 368-75.

[112] Smith L., Donlon B., Gupta M., Mohtai M., Das P. Carter D. et al. Effects of fluid-induced shear on articular chondrocyte morphology and metabolism in vitro. Orthop Res 1995;13: 824-31.

[113] Murphy C. and Polak J. Control of human articular chorocyte differentiation by reduced oxygen tension. J Cell Physiol 2004;199:451-9.

[114] Katopodi T., Tew S., Clegg P. and Hardingham T. The influence of donor and hypoxic conditions on the assembly of cartilage matrix by osteoarthritic human articular chondrocytes on Hyalograft matrices. Biomaterials 2009;4:53540.

[115] Jahr H., Gunes S., Kuhn A., Nebelung S. and Pufe T. Bioreactor-Controlled Physoxia Regulates TGF- $\beta$ signaling to alter extracellular matrix synthesis by human chondrocytes Int J Mol Sci 2019;20:1715.

[116] Wernike E., Li Z., Alini M. and Grad S. Effect of reduced oxygen tension and long-term mechanical stimulation on chondrocyte-polymer constructs. Cell Tissue Res 2008;331:473-83.

[117] Gunja N. and Athanasiou K. Additive and synergistic effects of bFGF and hypoxia on leporine meniscus cell-seeded PLLA scaffolds. J Tisue Eng Regen Med 2009;4:115-22.

[118] Meinert C., Schrobback K., Hutmacher D.W., Klein T.J. A novel bioreactor system for biaxial mechanical loading enhances the properties of tissueengineered human cartilage. Sci Rep 2017;7:1-14.

[119] Lee J.K., Huwe L.W., Paschos N., Aryaei A., Gegg C.A., Hu J.C., et al. Tension stimulation drives tissue formation in scaffold-free systems. Nat Mat 2017; $16: 864-73$

[120] Loeser R., Pacione C. and Chubinskaya S. The combination of insulin-like growth factor 1 and osteogenic protein 1 promotes increased survival of and matrix synthesis by normal and osteoarthritic human articular chondrocytes. Arthritis Rheum 2003;48: 2188-96.

[121] Yaeger P., Masi T., Buck J., Binette F., Tubo R. and McPherson J. Synergistic action of transforming growth factor- $\beta$ and insulin-like growth factor-I induces expressionof type II collagen and aggrecan genes in adult human articular chondrocytes. Expe. Cell Biol 1997;2:318-25

[122] Kabiri A., Esfandiari E., Hashemibeni B., Kazemi M., Mardani M. and Esmaeili A. Effects of FGF-2 on human adipose tissue derived adult stem cells morphology and chondrogenesis enhancement in Transwell culture. Biochem Biophys Res Commun 2012;424:234-8.

[123] Martin I., Wendt D. and Heberer M. The role of bioreactors in tissue engineering. Trends Biotechnol 2004;22:80-86.

[124] Grounds M.D. Obstacles and challenges for tissue engineering and regenerative medicine: Australian nuances. Clin Exp Pharmacol Physiol 2018;45:390400.

[125] Berthiaume F., Maguire T.J. and Yarmush M.L. Tissue Engineering and Regenerative Medicine: history, Progress, and Challenges. Annu Rev Chem Biomol Eng 2011;2:403-30.

[126] Huey D.J., Hu J.C. and Athanasiou K.A. Unlike bone, cartilage regeneration remains elusive. Science 2012;338:917-21.

[127] Mouser V.H.M., Abbadessa A., Levato R., Hennink W.E., Vermonden T., Gawlitta D. Development of a thermosensitive HAMA-containing bio-ink for the fabrication of composite cartilage repair constructs. Biofabrication 2017;9:015026.

[128] Moroni L., Thomas B., Burdick J.A., Maria C., Derby B., Forgacs G., et al. Biofabrication : a Guide to Technology and Terminology. Trends in Biotechnol 2018;36:1-19.

[129] Moroni L., Highley J.A.B.C., and Lee Yuya S.J. Biofabrication strategies for 3D in vitro models and regenerative medicine. Nat. Rev. Mater 2019;3:21-37

[130] Klein T.J., Malda J., Sah R.L., Hutmacher D.W. Tissue engineering of articular cartilage with biomimetic zones. Tissue Eng Part B Rev 2009;15:143-57.

[131] Schon B.S., Hooper G.J. and Woodfield T.B.F. Modular Tissue Assembly Strategies for Biofabrication of Engineered Cartilage. Ann Biomed Eng 2017;45:10014.

[132] Aspden R.M. Fibre reinforcing by collagen in cartilage and soft connective tissues. Proc. Royal Soc 1994;258:195-200.

[133] Cohen N.P., Foster R.J. and Mow V.C. Composition and dynamics of articular cartilage: structure, function, and maintaining healthy state. J Orthop Sports Phys Ther 1998;28:203-15.

[134] Pavlovich M.J., Hunsberger J. and Atala A. Biofabrication: a secret weapon to advance manufacturing, economies and healthcare. Trends Biotechnol 2016;34:1-2.

[135] Kang H.W., Lee S.J., Ko I.K., Kengla C., Yoo J., Atala A. A 3D bioprinting system to produce human-scale tissue constructs with structural integrity. Nat Biotechnol 2016;34:312-9.

[136] Peiffer Q., Ruijter M., van Duijn J., Crottet D., Malda J. and Castilho M. Melt electrowriting onto anatomically relevant biodegradable substrates: resurfacing a diarthrodrial joint. Mat Design 2020;195:10025.

[137] Xing D., Chen J., Yang J., Heng B.C., Ge Z., Lin J. Perspectives on Animal Models Utilized for the Research and Development of Regenerative Therapies for Articular Cartilage. Curr Mol Biol Rep. 2016;2:90-100.

[138] Zheng D., Dan Y., Yang S.H., Liu G.H., Shao Z.W., Yang C., et al. Controlled chondrogenesis from adipose-derived stem cells by recombinant transforming growth factor- $\beta 3$ fusion protein in peptide scaffolds. Acta Biomater 2015;11:191-203. 
[139] Haisch A, Groger A, Radke C, Ebmeyer J, Sudhoff H, Grasnick G, et al. Macroencapsulation of human cartilage implants: pilot study with polyelectrolyte complex membrane encapsulation. Biomaterials 2000;21:1561-6.

[140] Chu CR, Szczodry M, Bruno S. Animal models for cartilage regeneration and repair. Tissue Eng. Part B Rev. 2010;16:105-15.

[141] Tamai N, Myoui A. Hirao M, Kaito T, Ochi T, Tanaka J, et al. A new biotechnology for articular cartilage repair: subchondral implantation of a composite of interconnected porous hydroxyapatite, synthetic polymer (PLA-PEG), and bone morphogenetic protein-2 (rhBMP-2). Osteoarthr Cartil 2005;13:405-17.

[142] Ahern BJ, Parvizi J, Boston R, Schaer TP. Preclinical animal models in single site cartilage defect testing: a systematic review. Osteoarthr Cartil 2009; 17:705-13.

[143] Lin YX, Ding ZY, Zhou XB, Li ST, Xie M, Li ZZ, et al. In vitro and in vivo evaluation of the developed PLGA/HAp/Zein scaffolds for bone-cartilage interface regeneration. Biomed Environ Sci 2015;28:1-12.

[144] Gregory MH, Capito N, Kuroki K, Stoker AM, Cook JL, Sherman SL . A review of translational animal models for knee osteoarthritis. Arthritis 2012;2012:1-14

[145] Malda J, Benders KE, Klein TJ, Grauw JC, Hutmacher DW, Saris DB, et al. Comparative study of depth-dependent characteristics of equine and human osteochondral tissue from the medial and lateral femoral condyles. Osteoarthr Cartil 2012;20:1147-51.

[146] Moran CJ, Ramesh A, Brama PAJ, O’Byrne JM, O’Brien FJ, Levingstone TJ. The benefits and limitations of animal models for translational research in cartilage repair. J. Exp. Orthop 2016;3:1-12.

[147] European Medicines Agency GUIDELINE on human cell-based medicinal products; 2008. Available at URL: https://www.ema.europa.eu/en/documents/ scientific-guideline/guideline-human-cell-based-medicinal-products_en.pdf .

[148] European Medicines Agency Reflection paper on in-vitro cultured chondrocyte containing products for cartilage repair of the knee; 2009. Available at URL: https://www.ema.europa.eu/en/documents/scientific-guideline/ reflection-paper-vitro-cultured-chondrocyte-containing-products-cartilage-repair-knee_ en.pdf .

[149] European Medicines Agency Reflection paper on clinical aspects related to tissue engineered products; 2014. Available at URL: $\quad$ https://www.ema.europa.eu/en/documents/scientific-guideline/ reflection-paper-clinical-aspects-related-tissue-engineered-products_en.pdf .

[150] Vacanti JP, Langer R. Tissue engineering: the design and fabrication of living replacement devices for surgical reconstruction and transplantation. Lancet 1999;354:32-4.

[151] Langer R, Vacanti JP. Tissue Engineering. Educational Forum 1993;15:119.

[152] Hollister SJ. Scaffold engineering: a bridge to where? Biofabrication 2009;1:012001.

[153] Oberweis CV, Marchal JA, Ruiz EL, Martin PG. A worldwide overview of regulatory frameworks for tissue-based products. Tissue Eng Part B Rev 2020;26:181-96.

[154] Henkel J, Woodruff MA, Epari DR, Steck R, Glatt V, Dickinson IC, et al. Bone regeneration based on tissue engineering conceptions-a 21st century perspective. Bone Res 2013;1:216-48.

[155] Lee JK, Responte DJ, Cissell DD, Nolta JA, Athanassiou KA, et al. Clinical translation of stem cells: insight for cartilage therapies. Crit Rev Biotechnol 2015;34:89-100.

[156] Hollister SJ, Murphy WL. Scaffold translation: barriers between concept and clinic. Tissue Eng Part B Rev 2011;17:459-74.

[157] Ravichandran A, Liu Y, Teoh SH. Review: bioreactor design towards generation of relevant engineered tissues: focus on clinical translation. J Tissue Eng Regen Med 2018;12:e7-e22.

[158] Guilak F, Butler DL, Goldstein SA, Mooney D. Functional tissue engineering. Annals of the New York Academy of Sciences; 2002.

[159] Hurtig MB, Buschmann MD, Fortier LA, Hoemann CD, Hunziker EB, Jurvelin JS, et al. Preclinical studies for cartilage repair: recommendations from the international cartilage repair society. Cartilage 2011;2:137-52.

[160] Reinholz GG, Lu L, Saris DBF, Yaszemski MJ, O’Driscoll SW. Animal models for cartilage reconstruction. Biomaterials 2004;25:1511-21.

[161] Wei X, Messner K. Maturation-dependent durability of spontaneous cartilage repair in rabbit knee joint. J Biomed Mater Res 1999;46:539-48. 